\title{
Transition Temperature for Weakly Interacting Homogeneous Bose Gases
}

\author{
Frederico F. de Souza Cruz*, Marcus B. Pinto ${ }^{\dagger}$ \\ Departamento de Física, Universidade Federal de Santa Catarina, \\ 88040-900 Florianópolis, SC, Brazil \\ Rudnei O. Ramos ${ }^{\ddagger}$ \\ Department of Physics and Astronomy, Dartmouth College, Hanover, New Hampshire 03755-3528 \\ and ${ }^{(1)}$ Departamento de Física Teórica, Instituto de Física, \\ Universidade do Estado do Rio de Janeiro, 20550-013 Rio de Janeiro, RJ, Brazil
}

\begin{abstract}
We apply the non-perturbative optimized linear $\delta$ expansion method to the $O(N)$ scalar field model in three-dimensions to determine the transition temperature of a dilute homogeneous Bose gas. Our results show that the shift of the transition temperature, $\Delta T_{c} / T_{c}$, of the interacting model, compared with the ideal gas transition temperature, really behaves as $\gamma a n^{1 / 3}$ where $a$ is the s-wave scattering length and $n$ is the number density. For $N=2$ our calculations yield the value $\gamma=3.059$.
\end{abstract}

PACS number(s): 03.75.Fi, 05.30.Jp, 11.10.Wx

\section{INTRODUCTION}

The experimental realization of the Bose-Einstein condensation in dilute atomic gases has greatly stimulated an enormous number of theoretical studies in this field (for recent reviews on the theory and experiments, see for instance, Refs. [1,2]). Most of this interest comes from the fact that in these experiments a great deal of control can be achieved in almost every parameter of the system. Thus, experiments in dilute Bose gases provide a perfect ground to test numerous models and ideas, as for example those commonly used in quantum field theory, applied to non-relativistic systems. In particular, a theoretical study which has attracted some attention very recently is the determination of the behavior of the transition temperature in the presence of a repulsive interaction. This non trivial problem has been treated by different methods with different results. Taking a dilute Bose gas, with a repulsive interaction characterized by the scattering length parameter $a$, the dependence on $a$ for the difference between the critical temperature shift with and without interaction $\left(\Delta T_{c} / T_{c}=\left(T_{c}-T_{c}^{0}\right) / T_{c}\right)$ is a highly controversial point, be with respect to the functional dependence on $a$ or even regarding the sign. An early Hartree-Fock calculation [3] with a non-delta interaction gave

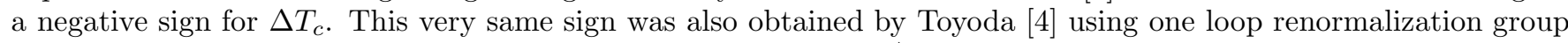
and obtaining a functional dependence on $a$ as $\Delta T_{c} / T_{c}=\gamma\left(a^{3} n\right)^{1 / 6}$, where $n$ is the density. More recently, Huang [5] obtained the same dependence for $\Delta T_{c} / T_{c}$, but with a positive constant $\gamma$. Grüter, Ceperley and Laloë [6], Holzmann and Krauth [7] and Holzmann, Grüter and Laloë [8] investigated the dependence of $\Delta T_{c}$ numerically using Monte Carlo methods. They obtained, in the low density limit, a dependence of the type $\Delta T_{c} / T_{c}=\gamma\left(a^{3} n\right)^{1 / 3}$ but with different values for $\gamma$. More recently the Monte Carlo technique has been again applied to this problem by Prokof'ev and Svistunov [9] and Arnold and Moore [10]. These authors, who obtain $\gamma=1.29 \pm 0.05$ and $\gamma=1.32 \pm 0.02$, respectively, claim that their results are more accurate than those obtained in Refs. [6] 8].

One of the reasons for the multitude of results and methods stem from the fact that at the transition temperature ordinary perturbation theory fails (due to infrared divergences) and we must resort to non-perturbative methods. Recently, there have been also used various non-perturbative methods to treat the problem of the transition temperature from an analytical way. For example, the authors in Ref. [11] perform a self-consistent calculation obtaining $\Delta T_{c} / T_{c}=\gamma a n^{1 / 3}$, with $\gamma \simeq 2.9$. The non-perturbative $1 / N$ method has been also used to determine the shift. Its leading order contribution has been evaluated by Baym, Blaizot and Zinn-Justin [12] who obtain $\Delta T_{c} / T_{c}=\gamma a n^{1 / 3}$, with $\gamma \simeq 2.33$ for $N=2$. Considering the next to leading order term, Arnold and Tomásik [13] determine a correction to this large- $N$ expansion, obtaining a value for $\Delta T_{c} / T_{c}$ which is $\sim 26 \%$ smaller.

The results obtained by Bijlsma and Stoof [14], who used renormalization group techniques, and Baym et al. [11] were compared with the temperature transition data in the Vycor- ${ }^{4} \mathrm{He}$ system by Reppy et al. [15]. Those experiments

\footnotetext{
*Email address: fred@fsc.ufsc.br

${ }^{\dagger}$ Email address: fsc1mep@fsc.ufsc.br

${ }^{\ddagger}$ Email address: rudnei@peterpan.dartmouth.edu. ${ }^{(1)}$ Permanent address.
} 
seem to give a somewhat larger value for the constant $\gamma$, as $\gamma \sim 5$ [15]. This value is close to $\gamma \simeq 4.66$ which is the one obtained in Ref. [14]. Nevertheless some authors argue that this system would not exactly correspond to a dilute Bose gas of hard spheres 13$]$.

In this paper we apply the non-perturbative linear $\delta$ expansion (or optimized perturbation theory) [16] (for earlier references see, e.g., Refs. [17 [19]) to an effective model for dilute homogeneous Bose gases. This approximation has been shown to be a powerful non-perturbative method, and sufficiently simple to use in very different applications, including the study of non-perturbative high temperature effects, as shown very recently in the context of finite temperature quantum field theory [20] as well as finite chemical potential [21]. The method also introduces an arbitrary mass parameter which prevents infrared divergence problems. Non-perturbative results are generated when one optimizes the theory with respect to this mass parameter.

The paper is organized as follows. We present the method in Sec. II illustrating with an application to the pure anharmonic oscillator which has many similarities with the model used here to describe dilute Bose gases. The interpolated version of an effective model for weakly interacting homogeneous Bose gases is obtained in Sec. III. Sec. IV is devoted to the perturbative evaluation of density related quantities. In Sec. V we present our non-perturbative results for the critical temperature shift comparing with results available in the literature. The conclusions are presented in Sec. VI.

\section{THE LINEAR $\delta$ EXPANSION}

The optimized linear $\delta$ expansion (LDE) is an alternative non-perturbative approximation which has been successfully used in a plethora of different problems in particle theory [16, 22, 24], quantum mechanics [18,25, 26], nuclear matter [27], lattice field theory [28] as well as for determining the equation of state for the Ising model [29]. One advantage of this method is that the selection and evaluation (including renormalization) of Feynman diagrams are done exactly as in perturbation theory using a very simple modified propagator which depends on an arbitrary mass parameter. The results are optimized with respect to this parameter at the end of the calculation. The standard application of the LDE to a theory described by some Lagrangian density $\mathcal{L}$ starts with an interpolation defined by

$$
\mathcal{L}_{\delta}=(1-\delta) \mathcal{L}_{0}(\eta)+\delta \mathcal{L}=\mathcal{L}_{0}(\eta)+\delta\left[\mathcal{L}-\mathcal{L}_{0}(\eta)\right],
$$

where $\mathcal{L}_{0}(\eta)$ is the Lagrangian density of a solvable theory which can contain arbitrary mass parameters $\eta$. The Lagrangian density $\mathcal{L}_{\delta}$ interpolates between the solvable $\mathcal{L}_{0}(\eta)$ (when $\delta=0$ ) and the original $\mathcal{L}$ (when $\delta=1$ ). To illustrate how the method works let us consider the anharmonic oscillator described by

$$
\mathcal{L}=\frac{1}{2}\left(\partial_{0} \phi\right)^{2}-\frac{1}{2} m^{2} \phi^{2}-\frac{\lambda}{4} \phi^{4}
$$

Following the interpolation prescription given by Eq. (2.1) one may choose

$$
\mathcal{L}_{0}(\eta)=\frac{1}{2}\left(\partial_{0} \phi\right)^{2}-\frac{1}{2} m^{2} \phi^{2}-\frac{1}{2} \eta^{2} \phi^{2}
$$

obtaining

$$
\mathcal{L}_{\delta}=\frac{1}{2}\left(\partial_{0} \phi\right)^{2}-\frac{1}{2} \Omega^{2} \phi^{2}-\delta \frac{\lambda}{4} \phi^{4}+\frac{\delta}{2} \eta^{2} \phi^{2},
$$

where $\Omega^{2}=m^{2}+\eta^{2}$. The general way the method works becomes clear by looking at the Feynman rules generated by $\mathcal{L}_{\delta}$. First, the original $\phi^{4}$ vertex has its original Feynman rule $-i 6 \lambda$ modified to $-i 6 \delta \lambda$. This minor modification is just a reminder that one is really expanding in orders of the artificial parameter $\delta$. Most importantly, let us look at the modifications implied by the addition of the arbitrary quadratic part. The original bare propagator,

$$
S(k)=i\left(k^{2}-m^{2}+i \epsilon\right)^{-1},
$$

becomes

$$
S(k)=i\left(k^{2}-\Omega^{2}+i \epsilon\right)^{-1}=\frac{i}{k^{2}-m^{2}+i \epsilon}\left[1-\frac{i}{k^{2}-m^{2}+i \epsilon}\left(-i \eta^{2}\right)\right]^{-1},
$$

indicating that the term proportional to $\eta^{2} \phi^{2}$ contained in $\mathcal{L}_{0}$ is entering the theory in a non-perturbative way. On the other hand, the piece proportional to $\delta \eta^{2} \phi^{2}$ is only being treated perturbatively as a quadratic vertex (of weight 
$\left.i \delta \eta^{2}\right)$. Since only an infinite order calculation would be able to compensate for the infinite number of $\left(-i \eta^{2}\right)$ insertions contained in Eq. (2.6), one always ends up with a $\eta$ dependence in any quantity calculated to finite order in $\delta$. Then, at the end of the calculation one sets the dummy parameter $\delta$ to unity (the value at which the original theory is retrieved) and fixes $\eta$ with the variational procedure known as the Principle of Minimal Sensitivity (PMS) [30] which requires that a physical quantity $P$ calculated perturbatively in powers of $\delta$ be evaluated at the point where it is less sensitive to variations of the arbitrary $\eta$. That is, one optimizes the perturbative calculation by requiring

$$
\left.\frac{\partial P(\eta)}{\partial \eta}\right|_{\bar{\eta}}=0 .
$$

This procedure gives $\bar{\eta}$ as a function of the original parameters, including the couplings, and generates non-perturbative results as shown in the numerous applications cited above.

As a warm-up for our application to the Bose gas problem we follow Bellet, Garcia and Neveu [31 evaluating the ground state energy density $\mathcal{E}$ and the vacuum expectation value $\left\langle\phi^{2}\right\rangle$ for the anharmonic oscillator. Other applications to this problem can be found in Refs. [18,25,26].

By taking $m=0$ in Eq. (2.2) one obtains the Lagrangian density for the pure anharmonic oscillator (PAO) which cannot be treated by ordinary perturbation theory. The exact result

$$
\mathcal{E}^{\text {exact }}=\lambda^{1 / 3} 0.420804974478 \ldots
$$

has been obtained by Bender, Olaussen and Wang [32], while Banerjee et al. [33] have obtained the exact result for $\left\langle\phi^{2}\right\rangle$

$$
\left\langle\phi^{2}\right\rangle^{\text {exact }}=\lambda^{-1 / 3} 0.456119955748 \ldots
$$

In quantum field theory, the ground state energy density is represented by vacuum to vacuum diagrams. The relevant contributions to $\mathcal{O}\left(\delta^{2}\right)$ are [31]

$$
\begin{aligned}
\mathcal{E}^{(2)}(\eta) & =-\frac{i}{2} \int_{-\infty}^{+\infty} \frac{d p}{2 \pi} \ln \left[p^{2}-\Omega^{2}\right]-\delta \frac{i}{2} \int_{-\infty}^{+\infty} \frac{d p}{2 \pi} \frac{\eta^{2}}{p^{2}-\Omega^{2}} \\
& -\delta \lambda \frac{3}{4}\left(\int_{-\infty}^{+\infty} \frac{d p}{2 \pi} \frac{1}{p^{2}-\Omega^{2}}\right)^{2}+\delta^{2} \frac{i}{4} \int_{-\infty}^{+\infty} \frac{d p}{2 \pi}\left[\frac{\eta^{2}}{p^{2}-\Omega^{2}}\right]^{2} \\
& +\delta^{2} \lambda \frac{3}{2} \int_{-\infty}^{+\infty} \frac{d p}{2 \pi} \frac{1}{p^{2}-\Omega^{2}} \int_{-\infty}^{+\infty} \frac{d p}{2 \pi} \frac{\eta^{2}}{\left(p^{2}-\Omega^{2}\right)^{2}} \\
& -\delta^{2} \lambda^{2} \frac{9}{4}\left(\int_{-\infty}^{+\infty} \frac{d p}{2 \pi} \frac{1}{p^{2}-\Omega^{2}}\right)^{2} \int_{-\infty}^{+\infty} \frac{d p}{2 \pi} \frac{1}{\left(p^{2}-\Omega^{2}\right)^{2}} \\
& -\delta^{2} \lambda^{2} \frac{3}{4} \int_{-\infty}^{+\infty} \frac{d p}{2 \pi} \int_{-\infty}^{+\infty} \frac{d q}{2 \pi} \int_{-\infty}^{+\infty} \frac{d l}{2 \pi}\left[\frac{1}{\left(p^{2}-\Omega^{2}\right)\left(q^{2}-\Omega^{2}\right)\left(l^{2}-\Omega^{2}\right)}\right. \\
& \left.\times \frac{1}{(p+q+l)^{2}-\Omega^{2}}\right]+O\left(\delta^{3}\right) .
\end{aligned}
$$

Note that the second, fourth and fifth contributions are due to the extra quadratic vertex, while all the others would also appear in an ordinary perturbative expansion to $\mathcal{O}\left(\lambda^{2}\right)$. Setting $m=0$ (PAO), evaluating the integrals and eliminating the divergent $\mathcal{O}\left(\delta^{0}\right)$ term one obtains

$$
\mathcal{E}^{(2)}(\eta)=\mathcal{E}^{(1)}(\eta)+\delta^{2} \frac{3 \lambda}{16 \eta^{2}}-\delta^{2} \frac{21 \lambda^{2}}{128 \eta^{5}}
$$

where

$$
\mathcal{E}^{(1)}(\eta)=\delta \frac{1}{4} \eta+\delta \frac{3 \lambda}{16 \eta^{2}}
$$

Good numerical results appear already at first order where the application of the PMS to $\mathcal{E}^{(1)}(\eta)$ yields $\mathcal{E}^{(1)}(\bar{\eta})=$ $\lambda^{1 / 3} 0.4290$ at $\bar{\eta}=(\lambda 1.5)^{1 / 3}$. To second order the authors in Ref. [31] obtain $\mathcal{E}^{(2)}(\bar{\eta})=\lambda^{1 / 3} 0.4210$ and then carry 
on improving this result to show convergence. The interested reader is referred to Ref. [31] for details concerning the optimization procedure (selection of roots, etc). Other proofs of convergence are given in Refs. [25] and [26].

Bellet, Garcia and Neveu also investigate the vacuum expectation value $\left\langle\phi^{2}\right\rangle$ and we discuss their results here because this physical quantity is particularly important for our application to bosonic condensates. The perturbative expansion for $\left\langle\phi^{2}\right\rangle$ can be obtained in different ways using standard quantum field theory methods. The authors in Ref. [31] prefer to do it from the perturbative expansion for $\mathcal{E}^{(\delta)}$ recalling that

$$
\left\langle\phi^{2}\right\rangle^{(\delta)}=2 \frac{\partial}{\partial \Omega^{2}} \mathcal{E}^{(\delta)} .
$$

Going to second order in $\delta$ they optimize this quantity in two different ways. First, by applying the PMS condition directly to $\left\langle\phi^{2}\right\rangle^{(2)}$ they obtain $\left\langle\phi^{2}\right\rangle^{(2)}=\lambda^{-1 / 3} 0.455758$. Next, they use the optimum values obtained by extremizing $\mathcal{E}^{(2)}$, getting $\left\langle\phi^{2}\right\rangle^{(2)}=\lambda^{-1 / 3} 0.454246$, showing that both approaches lead to results with same order of accuracy.

Still in the context of the anharmonic oscillator, Jones, Parkin and Winder in Ref. [34 have shown that the linear $\delta$ expansion applied to the calculation of dynamical evolution of $\left\langle\phi^{2}\right\rangle$, where the PMS is applied directly to this quantity, tracks the exact solution longer than any previous approximate methods used to study the same quantity, like Hartree-Fock or ordinary perturbation theory. This result also reinforces the correctness of our procedure of optimizing the density in this particular application to Bose condensates.

Before applying the $\delta$ expansion to the Bose-Einstein condensation problem let us clarify a few points regarding the method. Firstly, one could object to the fact that $\delta$ is formally treated as small during the actual calculation and finally set to unity at the end. However, we recall that the only role attributed to this dummy parameter is to label the orders so that one can keep track of the extra diagrams which arise from the quadratic vertex $\delta \eta^{2} \phi^{2}$.

Finally, one could ask how the LDE relates to other non-perturbative analytical methods such as the $1 / N$ expansion. To see that, let us consider the same model discussed above for the case where the dynamical variables are a set of $N$ scalar fields, $\phi^{a}(a=1, \ldots, N)$. Proceeding as before the $\delta$ expansion would give the following result for $\mathcal{E}$ at $\mathcal{O}(\delta)$,

$$
\mathcal{E}^{(1)}(\eta)=\delta N \frac{1}{4} \eta+\delta \frac{N(N+2)}{3} \frac{3 \lambda}{16 \eta^{2}} .
$$

The application of the PMS to this quantity gives

$$
\mathcal{E}^{(1)}(\bar{\eta})=\left[\frac{(N+2)}{3} \lambda\right]^{1 / 3} 0.4290
$$

at

$$
\bar{\eta}=[1.5(N+2) \lambda]^{1 / 3} .
$$

Higher order contributions bring more factors of $N$ (more loops) making the calculation meaningless if $N$ is very large. However, this particular limit can also be properly handled by the LDE provided one defines $g=N \lambda$ declaring that the large- $N$ limit will be studied with fixed $g$ [35]. Using $g=N \lambda$ in Eq. (2.16) one sees that, in the large- $N$ limit, $\bar{\eta}$ is of order $N^{0}$, in terms of which Eq. (2.14) gives that $\mathcal{E}^{(1)}(\bar{\eta})$ is of order $N$, exactly as the leading $1 / N$ result, as one can easily check.

An important result, proved in the context of the effective potential [36], shows that the LDE exactly reproduces large- $N$ results in any order in $\delta$ provided that one stays within the large- $N$ limit. Moreover, the LDE is sensitive to small- $N$ effects since these terms may appear in terms such as $N(N+2) \lambda$ in Eq. (2.14). In fact, Ref. [23] shows how small- $N$ effects are effectively taken into account by the LDE in the context of the $1+1$ dimensional GrossNeveu model at finite temperatures where the results nicely converge, order by order, towards the exact result set by Landau's theorem.

The formal relationship in between the LDE and $1 / N$ is investigated in detail in Refs. [23] and [36]. Here, we shall concern ourselves with the finite $N$ case only.

\section{THE INTERPOLATED MODEL FOR DILUTE HOMOGENEOUS BOSE GASES}

Let us start by considering the typical model that describes a gas of interacting boson particles, described by a complex scalar field $\psi$ with Lagrangian density given by 


$$
\begin{aligned}
\mathcal{L} & =\psi^{*}(\mathbf{x}, t)\left(i \frac{d}{d t}+\frac{1}{2 m} \nabla^{2}\right) \psi(\mathbf{x}, t)+\mu \psi^{*}(\mathbf{x}, t) \psi(\mathbf{x}, t) \\
& -\frac{1}{2} \int d^{3} x^{\prime} \psi(\mathbf{x}, t) \psi^{*}(\mathbf{x}, t) V\left(\mathbf{x}-\mathbf{x}^{\prime}\right) \psi\left(\mathbf{x}^{\prime}, t\right) \psi^{*}\left(\mathbf{x}^{\prime}, t\right)
\end{aligned}
$$

where $\mu$ is the chemical potential. Let us take the interatomic interaction potential as being the one for a hard sphere gas,

$$
V\left(\mathbf{x}-\mathbf{x}^{\prime}\right)=\frac{4 \pi a}{m} \delta\left(\mathbf{x}-\mathbf{x}^{\prime}\right)
$$

where $a$ is the $s$-wave scattering length.

We want to determine the deviation of the critical temperature $T_{c}$, of the interacting model, in relation to the critical temperature for Bose-Einstein condensation for a free gas, $T_{0}$, given by the usual expression

$$
T_{0}=\frac{2 \pi}{m}\left(\frac{n}{\zeta(3 / 2)}\right)^{\frac{2}{3}}
$$

where $n$ is the number density of the boson gas and $\zeta(3 / 2) \simeq 2.612$.

As discussed in Refs. [12] and [13], close to the critical point we can reduce (3.1) to an effective three-dimensional model for the zero Matsubara frequency modes (the static modes) of the fields $\psi$, given by the functional integration of the non-zero modes, obtaining an effective action defined by $\left(\beta^{-1}=T\right)$

$$
\int_{0}^{\beta} d \tau \int d^{3} x \mathcal{L}_{\mathrm{Eucl}}\left[\psi(\mathbf{x}, \tau), \psi^{*}(\mathbf{x}, \tau)\right] \rightarrow \beta \int d^{3} x \mathcal{L}_{\mathrm{eff}}\left[\psi(\mathbf{x}), \psi^{*}(\mathbf{x})\right],
$$

where $\mathcal{L}_{\text {Eucl }}$ is the Lagrangian density in Euclidean space $(\tau=i t$, as usual) and with the effective action for the static modes, $\int d^{3} x \mathcal{L}_{\text {eff }}$, being equivalent to a three-dimensional $O(2)$ field theory, defined by the action

$$
S=\int d^{3} x\left[\frac{1}{2}|\nabla \phi|^{2}+\frac{1}{2} r \phi^{2}+\frac{u}{4 !}\left(\phi^{2}\right)^{2}\right],
$$

where $\phi=\left(\phi_{1}, \phi_{2}\right)$ is related to the original real components of $\psi$ by $\psi_{1}=(m T)^{1 / 2} \phi_{1}$ and $\psi_{2}=(m T)^{1 / 2} \phi_{2}$ while $r$ and $u$ are given by

$$
r=-2 m \mu, \quad u=48 \pi a m T \text {. }
$$

By considering the usual interpolation prescription given by Eq. (2.1) we write

$$
S \rightarrow S_{\delta}=\delta S+(1-\delta) S_{0},
$$

where $S_{0}$ is quadratically (exactly solvable) in the fields.

One can choose

$$
S_{0}=\frac{1}{2}\left[|\nabla \phi|^{2}+R \phi^{2}\right]
$$

where $R=r+\eta^{2}$, obtaining

$$
S_{\delta}=\int d^{3} x\left[\frac{1}{2}|\nabla \phi|^{2}+\frac{1}{2} R \phi^{2}-\frac{\delta}{2} \eta^{2} \phi^{2}+\frac{\delta u}{4 !}\left(\phi^{2}\right)^{2}\right],
$$

with $\eta$ being an arbitrary parameter, with mass dimensions, which is fixed at a finite order in $\delta$ by the PMS condition, Eq. (2.7). Here we will optimize the physical quantity represented by $\left\langle\phi^{2}\right\rangle$ which, as we shall see, is directly related to the critical temperature shift $\Delta T_{c} / T_{c}$. Let us first define the density for the interacting case

$$
n=m T\left\langle\phi^{2}\right\rangle_{u}
$$

where, for the $O(N)$ symmetric model, $\left\langle\phi^{2}\right\rangle_{u}$ is expressed in terms of the three-dimensional dressed Green's function $G_{\delta}(p)$ as 


$$
\left\langle\phi^{2}\right\rangle_{u}=\sum_{i=1}^{N}\left\langle\phi_{i}^{2}\right\rangle_{u}=N \int \frac{d^{3} p}{(2 \pi)^{3}} G_{\delta}(p),
$$

where

$$
G_{\delta}(p)=\left[p^{2}+R-\delta \eta^{2}+\Sigma_{\delta}(p)\right]^{-1},
$$

and $\Sigma_{\delta}(p)$ is the $\phi$ field renormalized self energy which will be evaluated perturbatively in powers of $\delta$.

At the critical temperature the original system must exhibit infinite correlation length, which means that at $T_{c}$ and $\delta=1$ (the original theory), $G_{\delta}^{-1}(0)=0$. Then, one gets the relation

$$
r=-\Sigma_{\delta}(0)
$$

which is just the form of the Hugenholtz-Pines theorem. We must stress that the choice (3.9) respects the HugenholtzPines Theorem at all orders in $\delta$.

Now, by using the relation (3.13) in (3.11), one can write

$$
\left\langle\phi^{2}\right\rangle_{u}=\int \frac{d^{3} p}{(2 \pi)^{3}} \frac{N}{p^{2}+\eta^{2}}\left[1+\frac{\left(-\delta \eta^{2}\right)+\Sigma_{\delta}(p)-\Sigma_{\delta}(0)}{p^{2}+\eta^{2}}\right]^{-1}
$$

\section{EVALUATION OF $\left\langle\phi^{2}\right\rangle$ TO $\mathcal{O}\left(\delta^{2}\right)$}

Expanding the above expression, in powers of $\delta$, to $\mathcal{O}(\delta)$ one sees that the only contribution to the self energy is a momentum independent tadpole diagram which is canceled by the condition on $r$. Then, to order $\delta$, we obtain

$$
\left\langle\phi^{2}\right\rangle_{u}=\int \frac{d^{3} p}{(2 \pi)^{3}} \frac{N}{p^{2}+\eta^{2}}\left[1+\frac{\delta \eta^{2}}{p^{2}+\eta^{2}}\right]
$$

which is $u$ independent and cannot furnish non-perturbative results. At next order in $\delta$ the only momentum dependent contribution to the self energy comes from the two loop setting sun diagram, which is of order $\delta^{2}$. Then, we obtain

$$
\left\langle\phi^{2}\right\rangle_{u}=\int \frac{d^{3} p}{(2 \pi)^{3}} \frac{N}{p^{2}+\eta^{2}}\left[1+\frac{\delta \eta^{2}}{p^{2}+\eta^{2}}+\frac{\delta^{2} \eta^{4}}{\left(p^{2}+\eta^{2}\right)^{2}}-\frac{\Sigma_{s s}(p)-\Sigma_{s s}(0)}{p^{2}+\eta^{2}}\right]
$$

where $\Sigma_{s s}(p)$ represents the setting sun contribution to the self energy,

$$
\Sigma_{s s}(p)=-\frac{(N+2) u^{2} \delta^{2}}{18} \int \frac{d^{3} k}{(2 \pi)^{3}} \frac{d^{3} q}{(2 \pi)^{3}} \frac{1}{\left(k^{2}+\eta^{2}\right)\left(q^{2}+\eta^{2}\right)\left[(p+q+k)^{2}+\eta^{2}\right]} .
$$

Note that $\eta$ acts naturally as an infrared cutoff so we do not have to worry about these type of divergences. The first three terms in Eq. (4.2) represent one-loop diagrams with different powers of $\delta \eta^{2}$ insertions. We regularize all diagrams with dimensional regularization in arbitrary dimensions $d=3-2 \epsilon$ and carry the renormalization with the $\overline{M S}$ scheme. So the momentum integrals are replaced by

$$
\int \frac{d^{3} p}{(2 \pi)^{3}} \rightarrow \int_{p} \equiv\left(\frac{e^{\gamma_{E}} M^{2}}{4 \pi}\right)^{\epsilon} \int \frac{d^{d} p}{(2 \pi)^{d}}
$$

where $M$ is an arbitrary mass scale and $\gamma_{E} \simeq 0.5772$ is the Euler-Mascheroni constant. One then obtains the $\mathcal{O}\left(\delta^{2}\right)$ one loop contributions

$$
-\frac{N \eta}{4 \pi}+\frac{\delta}{2} \frac{N \eta}{4 \pi}+\frac{\delta^{2}}{8} \frac{N \eta}{4 \pi}+\mathcal{O}(\epsilon)
$$

where we have used the expression [37]

$$
\int_{p} \frac{1}{p^{2}+\eta^{2}}=-\frac{\eta}{4 \pi}\left[1+2 \epsilon\left(\ln \frac{M}{2 \eta}+1\right)+\mathcal{O}\left(\epsilon^{2}\right)\right]
$$


and its derivatives with respect to $\eta^{2}$ to determine Eq. (4.4). The setting sun self energy diagram, with zero external momentum, is given by (see, for example, Ref. [37])

$$
\left.\Sigma_{s s}(p)\right|_{p=0}=-\frac{(N+2)}{18} \frac{u^{2} \delta^{2}}{(8 \pi)^{2}}\left[\frac{1}{\epsilon}+4 \ln \frac{M}{2 \eta}+2+4 \ln \frac{2}{3}+\mathcal{O}(\epsilon)\right],
$$

from which one gets

$$
\int_{p} \frac{N}{\left(p^{2}+\eta^{2}\right)^{2}} \Sigma_{s s}(0)=-\frac{N(N+2)}{9} \frac{\delta^{2} u^{2}}{(8 \pi)^{3} \eta}\left[\frac{1}{2 \epsilon}+3 \ln \left(\frac{M}{2 \eta}\right)+1+2 \ln \frac{2}{3}+\mathcal{O}(\epsilon)\right] .
$$

The momentum dependent setting sun contribution can be written as

$$
\int_{p} \frac{N}{\left(p^{2}+\eta^{2}\right)^{2}} \Sigma_{s s}(p)=\frac{N(N+2) u^{2} \delta^{2}}{72} \frac{d}{d \eta^{2}} I_{\text {bask }},
$$

where [37]

$$
\begin{aligned}
I_{\text {bask }} & =\int_{p k q} \frac{1}{\left(p^{2}+\eta^{2}\right)\left(k^{2}+\eta^{2}\right)\left(q^{2}+\eta^{2}\right)\left[(p+q+k)^{2}+\eta^{2}\right]} \\
& =-\frac{\eta}{(4 \pi)^{3}}\left[\frac{1}{\epsilon}+6 \ln \frac{M}{2 \eta}+8-4 \ln 2+\mathcal{O}(\epsilon)\right] .
\end{aligned}
$$

We then obtain for Eq. (4.8)

$$
\int_{p} \frac{N}{\left(p^{2}+\eta^{2}\right)^{2}} \Sigma_{s s}(p)=-\frac{N(N+2)}{9} \frac{\delta^{2} u^{2}}{(8 \pi)^{3} \eta}\left[\frac{1}{2 \epsilon}+3 \ln \left(\frac{M}{2 \eta}\right)+1-2 \ln 2+\mathcal{O}(\epsilon)\right] .
$$

\section{THE TEMPERATURE SHIFT IN THE OPTIMIZED LINEAR $\delta$ EXPANSION}

Using Eqs. (4.4), (4.7) and (4.10) in (4.2), we determine $\left\langle\phi^{2}\right\rangle_{u}$ at order $\delta^{2}$. Note that all divergences in $\epsilon$ cancel and that at order $\delta^{2},\left\langle\phi^{2}\right\rangle_{u}$ is a finite quantity. One can now set $\delta=1$ and optimize $\left\langle\phi^{2}\right\rangle_{u}$ with the PMS. After that one sets $u=0$ in the optimized $\left\langle\phi^{2}\right\rangle_{u}$ obtaining the $\delta$ expansion result for the critical temperature shift [11 $[13$.

$$
\frac{\Delta T_{c}}{T_{c}} \simeq-\frac{2 m T_{0}}{3 n} \Delta\left\langle\phi^{2}\right\rangle=-\frac{2 m T_{0}}{3 n}\left[\left\langle\phi^{2}\right\rangle_{u}-\left\langle\phi^{2}\right\rangle_{0}\right],
$$

where $T_{0}$ is given by Eq. (3.3). At this stage it should be clear that it is preferable to optimize $\left\langle\phi^{2}\right\rangle_{u}$ rather than $\Delta\left\langle\phi^{2}\right\rangle$ because the latter quantity is less $\eta$ dependent. One then obtains

$$
\bar{\eta}= \pm\left[\frac{6(N+2) u^{2} \ln \frac{4}{3}}{(36 \pi)^{2}}\right]^{1 / 2}
$$

which leads to

$$
\left\langle\phi^{2}\right\rangle_{u}=\mp \frac{u N}{192 \pi^{2}}\left[6(N+2) \ln \frac{4}{3}\right]^{\frac{1}{2}} .
$$

In principle one could not single out one solution in favor of the other and we must be careful in choosing the appropriate one. Equation (5.3) implies that the optimized $\left\langle\phi^{2}\right\rangle_{0}$ vanishes no matter which sign is chosen. Now $\left\langle\phi^{2}\right\rangle_{0}$ represents the density (divided by a factor $m T$ ) in the absence of interactions, which turns out to be zero for the present effective theory. However, this is of no concern here since one is really interested in the difference $\left\langle\phi^{2}\right\rangle_{u}-\left\langle\phi^{2}\right\rangle_{0}$, represented by $\Delta T_{c}$. Also, one knows that the density of the interacting gas $\left(\left\langle\phi^{2}\right\rangle_{u}\right)$ should be smaller than that of the non interacting gas, which means that here one should have $\left\langle\phi^{2}\right\rangle_{u}<0$, which is achieved by selecting the positive $\bar{\eta}$. Using this in Eq. (5.1), we then get our final result, 


$$
\frac{\Delta T_{c}}{T_{c}} \simeq \frac{2 \pi}{\zeta(3 / 2)^{\frac{4}{3}}} \frac{N}{3}\left[6(N+2) \ln \frac{4}{3}\right]^{\frac{1}{2}} a n^{\frac{1}{3}} .
$$

Setting $N=2$ in the above expression yields

$$
\frac{\Delta T_{c}}{T_{c}} \simeq 3.059 a n^{\frac{1}{3}} .
$$

Using the $1 / N$ expansion Baym, Blaizot and Zinn-Justin [12] obtain $\Delta T_{c} / T_{c} \simeq 2.33 a n^{1 / 3}$ in the leading order while Arnold and Tomásik [13] obtain $\Delta T_{c} / T_{c} \sim 1.71 \mathrm{an}^{1 / 3}$ considering the next to leading order in the same approximation. Our result is closer to $\Delta T_{c} / T_{c} \simeq 2.9 a n^{1 / 3}$ obtained in Ref. [11] with a method which sums setting sun contributions in a self consistent way. These analytical results, including ours, are compared with the recent and earlier Monte Carlo estimates in Ref. [10]. Finally let us remark that the result given by Eq. (5.4) is valid only for finite $N$. This can be understood as follows. In a large $N$ study one would have to consider $u N$ as fixed (meaning that $u \sim N^{-1}$ ) and so, by taking $N$ large in Eq. (4.2), one sees that the setting sun diagrams of $\mathcal{O}\left(N^{0}\right)$ should be neglected since there are one loop diagrams of $\mathcal{O}(N)$. However, these terms are linear in $\eta$ as shown by their contribution, Eq. (4.4), and so the PMS does not give any meaningful result in this limit for the present model as opposed to the AO case. The difference arises mainly because momentum independent tadpole diagrams of $\mathcal{O}(N)$ are now being subtracted due to the Hugenholz-Pines theorem while the one loop momentum independent diagrams survive. Then, the PMS generates nontrivial results only by mixing diagrams which would belong to different orders in a standard large- $N$ application. To have a rough idea about what is being summed one can consider the second and fourth terms in Eq. (4.2) together with their integrated forms. Then it is clear that, apart from a numerical factor, the optimized $\bar{\eta}^{2}$ given by Eq. (5.2) behaves as the optimized $\Sigma_{s s}(p)-\Sigma_{s s}(0)$. One can then see that, to this order, the optimization dresses the simple propagator $\left(p^{2}+\eta^{2}\right)^{-1}$, present in Eq. (4.2), with setting sun features giving non-perturbative results which are compatible with the ones obtained in the self consistent summation of Ref. [11].

\section{CONCLUSIONS}

We conclude that our results for the critical temperature indeed reproduce the expected behavior obtained from other studies, which is $T_{c} \simeq T_{0}\left(1+\gamma a n^{1 / 3}\right)$. We obtain an analytical expression for the numerical coefficient $\gamma$ in terms of finite values of $N$. Our final numerical results are similar to the ones obtained with the self consistent summation 111 predicting that the numerical value of $\gamma$ is greater than the ones predicted by the $1 / N$ expansion at leading order 12. and next to leading order [13]. All these analytical results, including ours, have been compared with earlier and recent Monte Carlo results in Ref. [10]. It should be clear that the present calculation has been carried out to an order where only one two loop diagram contributes and so the quality of the approximation is hard to be inferred from a quantitative point of view. In fact, the purpose of the present application was just to introduce the method as a possible alternative to study the condensation problem. Nevertheless, one should remark that although carried out in a completely different fashion, our simple application seems to capture much of the features of the self consistent calculation performed by Baym et al. [11].

Also, our work does not exhaust the different ways in which this method can be implemented within this particular problem and the possibility of further improvements is still wide open. This could be achieved by investigating alternative forms of implementing the method within this model, including an investigation of the best quantity to be optimized, and/or by pushing the calculation to higher orders. It is possible that with more refinements this method will generate even better numerical results with the advantage, as shown in the paper, of being considerable simpler and easier to use than all previous methods used to determine the behavior of $T_{c}$.

Due to its simplicity and easy implementation, we believe that the optimized $\delta$ expansion can also be useful in other aspects of the theoretical study and understanding of the Bose condensation of dilute atomic gases, as determining the correct corrections to the energy spectrum, or in applications related to the recent studies of the dynamics of

the Bose-Einstein condensate formation [38]. The results of Ref. [34] are particularly motivating in the context of applying the optimized linear $\delta$ expansion also to dynamical problems. Finally, we point out that Bedingham and Evans [39] have successfully extended the present work to the ultra-relativistic case.

\section{ACKNOWLEDGMENTS}

The authors thank Philippe Garcia for his careful reading of Sec. II and Franck Laloë for interesting discussions regarding Bose-Einstein condensation as well as the method employed in this work. F.F.S.C. and M.B.P. were partially supported by CNPq-Brazil. R.O.R. was supported by CNPq-Brazil and SR2-UERJ. 
[1] F. Dalfovo, S. Giorgini and L. P. Pitaevskii, Rev. Mod. Phys. 71, 463 (1999).

[2] W. Ketterle, D. S. Durfee and D. M. Stamper-Kurn, in Bose-Einstein Condensation in Atomic Gases, Proceedings of the International School of Physics "Enrico Fermi", editors M. I. Inguscio, S. Stringari and C. E. Wieman (IOS Press, Amsterdam 1999).

[3] A. L. Fetter and J. D. Walecka, "Quantum Theory of Many-Particle Systems", (McGraw Hill, 1971), section 28.

[4] T. Toyoda, Ann. Phys. (N.Y.) 141, 154 (1982).

[5] K. Huang, Phys. Rev. Lett. 83, 3770 (1999).

[6] P. Grüter, D. Ceperley and F. Laloë, Phys. Rev. Lett. 79, 3549 (1997).

[7] M. Holzmann and W. Krauth, Phys. Rev. Lett. 83, 2687 (1999).

[8] M. Holzmann, P. Grüter and F. Laloë, Euro. Phys J. B 10, 739 (1999).

[9] N. Prokof'ev and B. Svistunov, cond-mat/0103149.

[10] P. Arnold and G. Moore, cond-mat/0103228.

[11] G. Baym, J.-P. Blaizot M. Holzmann, F. Laloë and D. Vautherin, Phys. Rev. Lett. 83, 1703 (1999).

[12] G. Baym, J.-P. Blaizot and J. Zinn-Justin, Europhys. Lett. 49, 150 (2000).

[13] P. Arnold and B. Tomásik, Phys. Rev. A 62, 063604 (2000).

[14] M. Bijlsma and H. T. C. Stoof, Phys. Rev. A 54, 5085 (1996).

[15] J. Reppy, B. Crooker, B. Hebral, A. Corwin, J. He and G. Zassanhaus, Phys. Rev. Lett. 84, 2060 (2000).

[16] A. Okopińska, Phys. Rev. D 35, 1835 (1987); A. Duncan and M. Moshe, Phys. Lett. B 215, 352 (1988).

[17] V. I. Yukalov, Mosc. Univ. Phys. Bull. 31, 10 (1976).

[18] R. Seznec and J. Zinn-Justin, J. Math. Phys. 20, 1398 (1979).

[19] J. C. LeGuillou and J. Zinn-Justin, Ann. Phys. (N.Y.) 147, 57 (1983).

[20] M. B. Pinto and R. O. Ramos, Phys. Rev. D 60, 105005 (1999); ibid. D 61, 125016 (2000).

[21] H. F. Jones and P. Parkin, Nucl. Phys. B 594, 518 (2001).

[22] A. Okopińska, Phys. Rev. D 36, 2415 (1987).

[23] S. K. Gandhi and M. B. Pinto, Phys. Rev D 49, 4258 (1994).

[24] M. B. Pinto, Phys. Rev D 50, 7673 (1994).

[25] I. R. C. Buckley, A. Duncan and H. F. Jones, Phys. Rev. D 47, 2554 (1993); A. Duncan and H. F. Jones, ibid. D 47, 2560 (1993); C. M. Bender, A. Duncan and H. F. Jones, ibid. D 49, 4219 (1994); C. Arvanitis, H. F. Jones and C. S. Parker, Phys. Rev. D 52, 3704 (1995).

[26] R. Guida, K. Konishi and H. Suzuki, Ann. Phys. (N.Y.) 249, 109 (1996).

[27] G. Krein, D. P. Menezes and M. B. Pinto, Phys. Lett. B 370, 5 (1996); G. Krein, R. S. M. Carvalho, D. P. Menezes, M. Nielsen, M. B. Pinto, Eur. Phys. J. A 1, 45 (1998).

[28] T. S. Evans, H. F. Jones and A. Ritz, Nucl. Phys. B 517, 599 (1998).

[29] R. Guida and J. Zinn-Justin, Nucl. Phys. B 489, 626 (1997).

[30] P. M. Stevenson, Phys. Rev. D 232916 (1981).

[31] B. Bellet, P. Garcia and A. Neveu, Int. J. of Mod. Phys A 11, 5587 (1997); ibid. A 11, 5607 (1997).

[32] C. M. Bender, K. Olausen and P. S. Wang, Phys. Rev. D 161780 (1977).

[33] K. Banerjee, S.P. Bhatnager, V. Choudry and S.S. Kanwal, Proc. R. Soc. London A 360575 (1978).

[34] H. F. Jones, P. Parkin and D. Winder, hep-th/0008069.

[35] S. Coleman, "Aspects of Symmetry" (Cambridge University Press, Cambridge, 1988).

[36] S. K. Gandhi, H. F. Jones and M. B. Pinto, Nucl. Phys. B359 429 (1991).

[37] E. Braaten and A. Nieto, Phys. Rev. D51, 6990 (1995).

[38] D. G. Barci, E. S. Fraga and R. O. Ramos, Phys. Rev. Lett. 85, 479 (2000).

[39] D. J. Bedingham and T. S. Evans, hep-ph/0011286. 